\title{
Methylprednisolone Modulates Intracranial Pressure in the Brain Cellular Edema Induced by Water Intoxication
}

\author{
P. KOZLER ${ }^{1}$, D. MAREŠOVÁ ${ }^{1}$, J. POKORNÝ ${ }^{1}$ \\ ${ }^{1}$ Institute of Physiology, First Faculty of Medicine, Charles University, Prague, Czech Republic
}

Received March 20, 2017

Accepted October 6, 2017

\section{Summary}

Continuous monitoring of the intracranial pressure (ICP) detects impending intracranial hypertension resulting from the impaired intracranial volume homeostasis, when expanding volume generates pressure increase. In this study, cellular brain edema (CE) was induced in rats by water intoxication (WI). Methylprednisolone (MP) was administered intraperitoneally (i.p.) before the start of CE induction, during the induction and after the induction. ICP was monitored for $60 \mathrm{~min}$ within $20 \mathrm{~h}$ after the completion of the $\mathrm{CE}$ induction by fibreoptic pressure transmitter. In rats with induced $C E$, ICP was increased (Mean $\pm S E M$ : $14.25 \pm 2.12)$ as well as in rats with MP administration before the start of CE induction (10.55 \pm 1.27$)$. In control rats without CE induction (4.62 \pm 0.24$)$ as well as in rats with MP applied during CE induction $(5.52 \pm 1.32)$ and in rats with MP applied after the end of CE induction $(6.23 \pm 0.73)$ ICP was normal. In the last two groups of rats, though the CE was induced, intracranial volume homeostasis was not impaired, intracranial volume as well as ICP were not increased. It is possible to conclude that methylprednisolone significantly influenced intracranial homeostasis and thus also the ICP values in the model of cellular brain edema.

\section{Key words}

Intracranial pressure monitoring - Water intoxication • Brain edema • Methylprednisolone

\section{Corresponding author}

J. Pokorný, Institute of Physiology, First Faculty of Medicine, Charles University, Albertov 5, Prague 2, 128 00, Czech Republic. E-mail: jaroslav.pokorny@lf1.cuni.cz

\section{Introduction}

Intracranial hypertension threatens the life in two ways - by compression of the vital centers of the brain cones or due to the loss of brain perfusion pressure. Intracranial hypertension can be caused by impaired volume homeostasis in the intracranial space (MonroeKellie doctrine), when the increasing volume generates increasing pressure. The increased volume may result from focal or diffuse intracranial process. Intracranial pressure (ICP) increases gradually depending on the degree of exhaustion of physiological compensatory mechanisms. Relations between increasing volume, the state of compensation and the increasing pressure are described in the form of pressure-volume curve (Langfitt et al. 1964). Normal value of the intracranial pressure in humans is between $5-15 \mathrm{~mm} \mathrm{Hg}$, depending on the position of the body, pressure higher than $15 \mathrm{~mm} \mathrm{Hg}$ signifies increased intracranial pressure and the values over $20 \mathrm{~mm} \mathrm{Hg}$ indicate intracranial hypertension. Details to the clinics of the intracranial pressure and methods of its monitoring are referred in details in review from 2015 (Gupta G: Intracranial pressure monitoring http://emedicine.medscape.com/article/1829950overview).

The only way to detect impending intracranial hypertension is the continuous monitoring of ICP. It was introduced into the clinical practice by Lundberg (Lundberg et al. 1965); the first clinical study on the importance of ICP monitoring during the management of the traumatic brain injury (TBI) was described by Becker (Becker et al. 1977). At present the ICP monitoring in patients with severe TBI represents an integral part of the guidelines (Brain Trauma Foundation 2007). For continuous monitoring of ICP in rodent experimental models, two principles are used. The first is a fibreoptic pressure transmitter for short-term (hours) monitoring in anesthetized and fixed animals, which since its deployment in the nineties of the previous century (Crutchfield et al. 1990, Zwienenberg et al. 1999) has 
been technologically developed (Murtha et al. 2012). The second method allows the long-term (days to weeks) ICP monitoring in freely moving animals on the principle of telemetry transmitters (Hiploylee and Colbourn 2014, Udall et al. 2014).

One diffusion process which increases the intracranial volume together with intracranial pressure is the brain edema. Our studies have been focused on one type of brain edema. According to the classification (Klatzo 1967, Go 1997, Kimlberg 1995, Liang et al. 2007) it belongs in terms of pathophysiology to cytotoxic edema, in terms of localization of the accumulating fluids it is the diffuse intracellular swelling. This type of cerebral edema occurs in clinical practice very frequently because of cerebral ischaemia or diffuse brain injuries. Contemporary medicine does not know any causal treatments that would prevent this type of brain swelling from the progressing. There is only a symptomatic treatment - osmotic, diuretic, and in extreme cases, surgical (decompressive craniotomy). In experimental models for studying cellular swelling, the routinely used method is water intoxication (Silver et al. 1999, Manley et al. 2000, Vajda et al. 2000). For many years, the objective of experimental studies was to find a method to prevent the induction or the development of swelling in its initial stages (Bullock et al. 1999). From this perspective, it appears promising the use of neuroprotective effect of certain substances that modulate permeability of cytoplasmic membranes, e.g. methylprednisolone.

\section{Methods}

For the experiment, adult male rats of the Wistar strain were used (weight $350-450 \mathrm{~g}$ ) and treated in accordance with the current Guidelines for the treatment of laboratory animals (EU Guidelines 86/609/EEC).

The group of 40 animals was divided in the following groups of eight rats: C (control group, untreated animals), WI (animals with induced cellular edema CE), WIMP (methylprednisolone MP injected during $\mathrm{CE}$ induction), WI + MP (MP was administered after the completion CE induction), MP + WI (MP was administered before the beginning of $\mathrm{CE}$ induction).

CE was induced by water intoxication in the group WI by injection of distilled water (DW) in a total amount corresponding to $20 \%$ of body weight intraperitoneally (i.p.) in three consecutive doses over $24 \mathrm{~h}$ with simultaneous administration of desmopressin.
Each sub-dose represented one third of the total dose $0.032 \mathrm{mg} / \mathrm{kg}$ (desmopressin (1-desamino-8-D-arginine vasopressin) (OCTOSTIM $\AA$, Ferring). Desmopressin is an antidiuretic hormone which potentiates the effect of hyperhydration by inducing hyponatremia.

Methylprednisolone (MP) was administered intraperitoneally in the dose of $100 \mathrm{mg} / \mathrm{kg}$ (Solu-Medrol®, Pfizer). This dose was derived from its proven neuroprotective effect in other studies (Park 1998, Slivka and Murphy 2001, Kozler et al. 2011, Kozler and Pokorný 2012). In the group MPWI, MP was given at the same time as the injection of water in the amount of one third of the total dose, together with similar dose of desmopressin (see Method CE induction). In the group of $\mathrm{WI}+\mathrm{MP}$, MP was given in a single dose of $100 \mathrm{mg} / \mathrm{kg}$ i.p. along with the last partial dose of desmopressin and DW. In the group of MP + WI, MP was injected in a single dose of $100 \mathrm{mg} / \mathrm{kg}$ i.p. with the first partial dose of DW and desmopressin. Monitoring of ICP in the WI group and in groups with MP administration begun $20 \mathrm{~h}$ after the completion of $\mathrm{CE}$ induction. Interval of $20 \mathrm{~h}$ excluded the effect of the MP's pharmacokinetics. Methylprednisolone is metabolized in the liver with a mean elimination half-life in the range from 1.8 to $5.2 \mathrm{~h}$.

Continuous monitoring of intracranial pressure (ICP) by fibrooptic system OPSENS MEDICAL $^{\mathrm{TM}}$ : Spontaneously breathing rats under the inhalation anesthetic isoflurane (Forane ${ }^{\circledR}$, AbbVie Ltd.) in concentration of 2 volume $\%$ underwent in the prone position the longitudinal incision of the skin and subcutaneous tissue in the midline of the head, free galea aponeurotica was dissected, and the scull was trephined $3 \mathrm{~mm}$ lateral to the midline at right and $3 \mathrm{~mm}$ frontally to bregma, dura was opened by incision and the microchip with pressure sensor connected to fibrooptic transmitter was introduced into the depth of $3 \mathrm{~mm}$ intraparenchymally. Transmitter was wired to a digital monitor and pressure analyser (part of software PC) for a continuous monitoring of the absolute values of ICP with current ICP curves on the PC monitor. After 60 min of the monitoring the microchip was removed, the durotomy and trepanopunction were sealed with Spongostan (SPONGOSTAN ${ }^{\mathrm{TM}}$, Ferrosan), subcutaneous tissue and the scalp were sutured. After completion of inhalation anesthesia, the rat spontaneously evoked at side position in about 25-30 min.

Software for ICP monitoring evaluated for each animal in interval of $60 \mathrm{~min}$ (Table 1). 
The results of all measurements were statistically evaluated using the GraphPad Prism program (parametric ANOVA and nonparametric Kruskal-Wallis test, the statistical significance was set at $5 \%$ ).

Table 1. Example of 60-minute interval of ICP monitoring in one animal.

\begin{tabular}{|c|c|c|c|c|c|c|}
\hline Unit & Minimum & Timestamp (s) & Maximum & Timestamp (s) & Mean & SEM \\
\hline $\mathrm{mm} \mathrm{Hg}$ & 2.2 & 10328 & 5.7 & 5396 & 4.308 & 0.6919 \\
\hline
\end{tabular}

Minimum and Timestamp (s) - the lowest recorded ICP value and its duration in seconds; Maximum and Timestamp (s) - the highest recorded ICP value and its duration in seconds; Mean - average ICP value during 60 min of monitoring; SEM (Standard Error of Mean).

\section{Results}

Mean ICP values ( \pm SEM) of eight animals in each group were as follows: $\mathrm{C}: 4.62 \pm 1.24$, WI: 14.25 \pm 2.12 , WIMP: 5.52 \pm 1.32 , WI + MP: $6.23 \pm 0.73$, MP + WI 10.55 \pm 1.27 .

ICP values in the individual groups significantly differed (Figs 1 and 2).

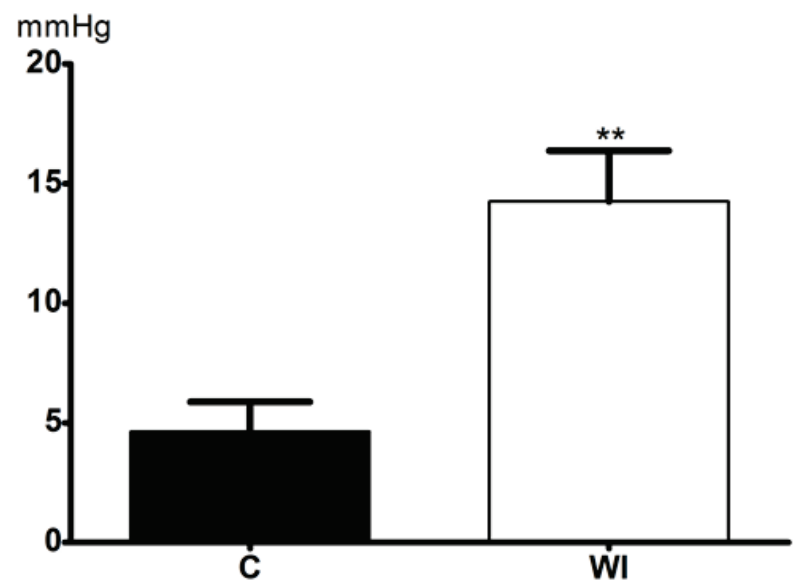

Fig. 1. $x$-axis: ICP value in $\mathrm{mm} \mathrm{Hg}, \mathrm{y}$-axis: $\mathrm{C}-$ control group of intact rats, WI - rats with induced $\mathrm{CE} ; * * p<0.1$.

In rats of the group with $\mathrm{CE}$, ICP values were significantly higher than those in the group of intact animals. As water intoxication increases the water content in the brain (differences in the dry and wet weight - Kozler et al. 2013 and differences in density in CT analysis - Kozler and Pokorný 2014), the measured values indicate the developing impairment of brain homeostasis.

Rats in group where MP was applied during CE induction and those of the group with MP applied after the completion of $\mathrm{CE}$ induction had significantly lower ICP values than the rats with induced CE without any further treatment. MP appears to have a neuroprotective effect, which was also demonstrated in the cytoplasmic membrane permeability changes (Kozler and Pokorný 2004) and the integrity of myelin (Kozler et al. 2011, Kozler and Pokorný 2012). These results revealed normal brain volume homeostasis, with the volume and ICP corresponding to normal levels of a rat in horizontal position (C: 4.62 \pm 1.24 , WIMP: $5.52 \pm 1.32$, WI + MP: 6.23 \pm 0.73$)$.

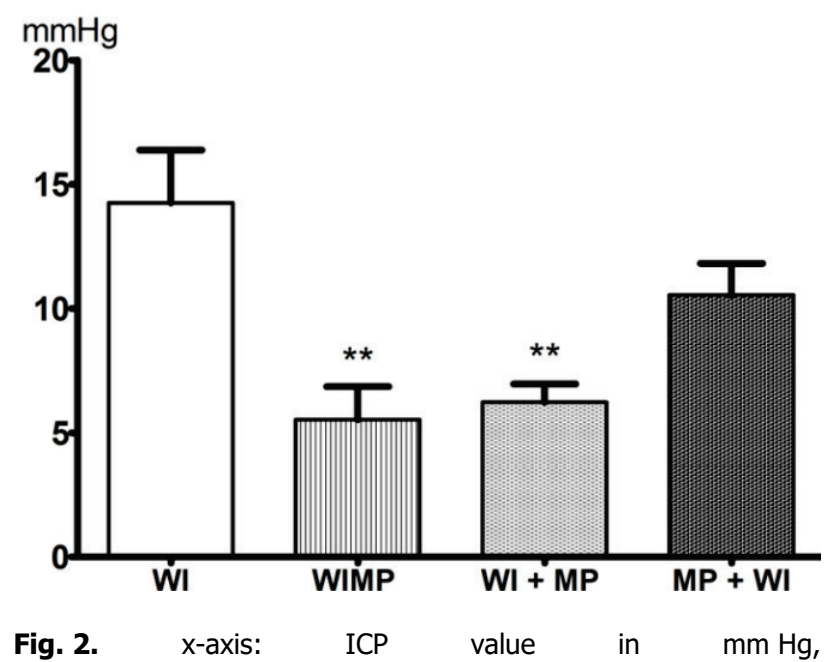
$y$-axis: WI - rats with induced CE; WIMP - MP was administered during the CE induction; WI + MP - MP was given after the completion of CE induction; MP + WI - MP was administered before the CE induction. Significance $(p<0.1)$ between the group with water intoxication only and individual MP administered groups is given $* *$.

In rats with MP applied before the CE induction, ICP values did not differ from the group of rats with induced CE. ICP value was in lying rats increased (MP + WI: 10.55 \pm 1.27$)$. This result indicates an impairment of brain volume homeostasis, with the increased intracranial volume and increased intracranial pressure. It can be concluded that in this experimental design effect of MP failed to develop. 


\section{Discussion}

The primary neuroprotective effect of methylprednisolone (MP) is attributed to its antioxidative ability which protects membrane lipids from peroxidation and against all subsequent adverse effects like changes in membrane fluidity or changes in the activity of membrane proteins (ion channels, transporters, enzymes). As the reactive oxygen species affect also other cellular systems (mitochondria, intracellular enzymes and co-factors, systems of transcription and translation) and they can thus alter various parameters of cell activity or induce the cell death by apoptosis, antioxidative ability of methylprednisone could interfere with the pathogenic process in nerve cells at different levels (Faden and Salzman 1992, Hall 1992, Hall 1993). This phenomenon is employed in the standard treatment for spinal cord injury (Bracken et al. 1997). MP in the damaged central nervous system can induce its positive effect by several mechanisms (Park 1998): 1) protective effects on axonal integrity against various degenerative influences by preservation of lysosomal integrity and membrane-bound enzymes, 2) enhancement of blood flow and preservation of microcirculatory pathways, 3 ) inhibition effect on the production of thromboxane and prostaglandin, 4) reinforcement of the capillary endothelial membranes maintenance, 5) reducing the permeability of the bloodbrain barrier, and 6) activation of anti-inflammatory processes. Regardless such spectrum of positive effects, corticotherapy is recommended only in selected and specific brain pathologies.

Methylprednisolone is a synthetic steroid with four times higher glucotropic and one fifth of the mineralotropic action of cortisol (hydrocortisone). Being a steroid, MP is of a lipophilic nature and is only weakly soluble in water. To be distributed in the body fluids it has to be in the form of ester methylprednisolone sodium succinate (MPSS). MPSS is not stabile and due to activity of hepatic esterases, MP is released and subsequently bound to plasma proteins in the ratio of 40 to $60 \%$. Contrary to the free liposoluble MP, the high-molecular complexes cannot cross the blood-brain barrier. From the total MPSS administered intravenously or intraparietally, only about one half can cross the blood brain barrier (Hall 1992).

High molecular weight substance such as MP bound to the albumin (MP>50 kDa) can be incorporated into the brain, either by a clinically used technique of osmotic opening of the BBB (Rapoport 2000, Kroll and Neuwelt 1998), or during the induced cellular edema by water intoxication.

Cellular edema induced in our study by WI (hyperhydration and hyponatremia) brings about an osmotic imbalance at the cell membrane followed by intracellular flow of sodium and simultaneous accumulation of water. This primary effect will initiate a cascade of processes leading to the subsequent increase of BBB permeability. The cascade includes: the loss of calcium and potassium homeostasis (Siesjo 1993, Kimelberg 1995, Barzó et al. 1997), release of excitotoxic amino acids (Bullock et al. 1994, Kimelberg 1995, Barzó et al. 1997), release of free oxygen radicals (Kontos 1989, Kimelberg 1995, Barzó et al. 1997), and induction of intracerebral tissue acidosis (Siesjo et al. 1993, Kimelberg 1995, Barzó et al. 1997).

When MP is administered during or at the end of the $\mathrm{CE}$ induction (together with the last sub-dose of desmopressin and DW), as it was in the experimental groups WIMP and WI + MP, MP penetrates into the brain due to increase permeability of BBB. It can therefore affect the permeability of the cytoplasmic membrane, block the intracellular water accumulation and prevent the development of cellular edema already at the initial stage. This effect cannot develop when MP is administered before the CE induction (together with the first sub-dose of DW and desmopressin), as it was in the experimental group MP + WI. In such arrangement, MP cannot penetrate into the brain in sufficient amounts because the BBB permeability is not increased during the cellular edema. ICP values during the 60-minute monitoring proved those MP effects. In the groups WIMP and $\mathrm{WI}+\mathrm{MP}$ intracranial volume was not increased, volume homeostasis was not violated and ICP values were normal. In the group of MP + WI the intracranial volume homeostasis failed to control the intracranial volume and ICP values were increased.

On the basis of this study we can conclude that the neuroprotective effect of MP can significantly affects the value of ICP during the cellular brain edema induced by water intoxication.

\section{Conflict of Interest}

There is no conflict of interest.

\section{Acknowledgements}

Supported with grant Progres Q35. 


\section{References}

BARZÓ P, MARMAROU A, FATOUROS P, HAYASAKI K, CORWIN F: Contribution of vasogenic and cellular edema to traumatic brain swelling measured by diffusion-weighted imaging. J Neurosurg 87: 900-907, 1997.

BECKER DP, MILLER JD, WARD JD, GREENBERG RP, YOUNG HF, SAKALAS R: The outcome from severe head injury with early diagnosis and intensive management. J Neurosurg 47: 491-502, 1977.

BRACKEN MB, SHEPARD MJ, HOLFORD TR: Administration of methylprednisolone for 24 or 48 hours or tirilizad mesylate for 48 hours in the treatment of acute spinal cord injury. JAMA 277: 1597-1604, 1997.

BRAIN TRAUMA FOUNDATION: Guidelines for the management of severe traumatic brain injury. 3rd Edition. J Neurotrauma 24 (Suppl 1): 2007, 106 p.

BULLOCK R, ZAUNER A, TSUJI O: Excitatory amino acid release after severe human head trauma: effect of intracranial pressure and cerebral perfusion pressure changes. In: Intracranial Pressure IX. NAGAI H, KAMIYA K, ISHII S (eds), Springer, Tokyo, 1994, pp 264-267.

BULLOCK MR, LYETH BG, MUIZELAAR JP: Current status of neuroprotection trials for traumatic brain injury: lessons from animal models and clinical studies. Neurosurgery 45: 207-220, 1999.

CRUTCHFIELD JS, NARAYAN RK, ROBERTSON CS, MICHAEL LH: Evaluation of a fiberoptic intracranial pressure monitor. J Neurosurg 72: 482-487, 1990.

FADEN AI, SALZMAN S: Pharmacological strategies in CNS trauma. Trends Pharmacol Sci 13: 29-35, 1992.

GO KG: The normal and pathological physiology of brain water. Adv Tech Stand Neurosurg 23: 47-142, 1997.

HALL ED: The neuroprotective pharmacology of methylprednisolone. J Neurosurg 76: 13-22, 1992.

HALL ED: The role of oxygen radicals in traumatic injury: clinical implications. J Emerg Med 11: 31-36, 1993.

HIPLOYLEE C, COLBOURNE F: Intracranial pressure measured in freely moving rats for days after intracerebral hemorrhage. Exp Neurol 255: 49-55, 2014.

http://emedicine.medscape.com/article/1829950-overview. 2015.

KIMELBERG HK: Current concepts of brain edema. J Neurosurg 83: 1051-1095, 1995.

KLATZO I: Presidental address. Neuropathological aspects of brain edema. J Neuropathol Exp Neurol 26: 1-14, 1967.

KONTOS HA: Oxygen radicals in CNS damage. Chem Biol Interact 72: 229-255, 1989.

KOZLER P, POKORNÝ J: Effects of intracarotid injection of methylprednisolone on cellular oedema after osmotic opening of the blood-brain barrier in rats. Prague Med Rep 105: 279-290, 2004.

KOZLER P, RILJAK V, POKORNÝ J: Methylprednisolone reduces axonal impairment in the experimental model of brain oedema. Neuro Endocrinol Lett 32: 831-835, 2011.

KOZLER P, POKORNY J: Effect of methylprednisolone on the axonal impairment accompanying cellular brain oedema induced by water intoxication in rats. Neuro Endocrinol Lett 33: 782-786, 2012.

KOZLER P, RILJAK V, POKORNÝ J: Both water intoxication and osmotic BBB disruption increase brain water content in rats. Physiol Res 62 (Suppl 1): S75-S80, 2013.

KOZLER P, POKORNY J: CT density decrease in water intoxication rat model of brain oedema. Neuro Endocrinol Lett 35: 608-612, 2014.

KROLL RA, NEUWELT EA: Outwitting the blood-brain barrier for therapeutic purposes: osmotic opening and other means. Neurosurgery 42: 1083-1100, 1998.

LANGFITT TW, WEINSTEIN JD, KASSELL NF, SIMEONE FA: Transmission of increased intracranial pressure. I. Within the craniospinal axis. J Neurosurg 21: 989-997, 1964.

LIANG D, BHATTA S, GERZANICH V, SIMARD JM: Cytotoxic edema: mechanisms of pathological cell swelling. Neurosurg Focus 22: E2, 2007.

LUNDBERG N, TROUPP H, LORIN H: Continuous recording of the ventricular-fluid pressure in patients with severe acute traumatic brain injury. A preliminary report. J Neurosurg 22: 581-590, 1965.

MANLEY GT, FUJIMURA M, MA T, NOSHITA N, FILIZ F, BOLLEN AW, CHAN P, VERKMAN AS: Aquaporin4 deletion in mice reduces brain edema after acute water intoxication and ischemic stroke. Nat Med 6: 159-163, 2000.

MURTHA L, MCLEOD D, SPRATT N: Epidural intracranial pressure measurement in rats using a fiber-optic pressure transducer. J Vis Exp 62: pii: 3689, 2012. 
PARK CO: The effects of methylprednisolone on prevention of brain edema after experimental moderate diffuse brain injury in rats: comparison between dosage, injection time, and treatment methods. Yonsei Med J 39: 395-403, 1998.

RAPOPORT SI: Osmotic opening of the blood-brain barrier: principles, mechanism, and therapeutic applications. Cell Mol Neurobiol 20: 217-230, 2000.

SIESJO BK: Basic mechanisms of traumatic brain damage. Ann Emerg Med 22: 959-969, 1993.

SIESJO BK, KATSURA K, MELLEGARD P: Acidosis-related brain damage. Prog Brain Res 96: 23-48, 1993.

SILVER SM, SCHROEDER BM, BERNSTEIN P, STERNS RH: Brain adaptation to acute hyponatremia in young rats. Am J Physiol 276: R1595-R1599, 1999.

SLIVKA PA, MURPHY EJ: High-dose methylprednisolone treatment in experimental focal cerebral ischemia. Exp Neurol 167: 166-172, 2001.

ULDALL M, JUHLER M, SKJOLDING AD, KRUUSE C, JANSEN-OLESEN I, JENSEN R: A novel method for long-term monitoring of intracranial pressure in rats. J Neurosci Methods 227: 1-9, 2014.

VAJDA Z, PROMENEUR D, DÓCZI T, SULYOK E, FRØKIAER J, OTTERSEN OP, NIELSEN S: Increased aquaporin-4 immunoreactivity in rat brain in response to systemic hyponatremia. Biochem Biophys Res Commun 270: 495-503, 2000.

ZWIENENBERG M, GONG QZ, LEE LL, BERMAN RF, LYETH BG: ICP monitoring in the rat: comparison of monitoring in the ventricle, brain parenchyma, and cisterna magna. J Neurotrauma 16: 1095-1102, 1999. 